\title{
Ultrasonic fatigue experiments with biaxial cruciform specimens
}

\author{
Pedro R. da Costa ${ }^{1}$, Diogo Montalvão ${ }^{2}$, Manuel Freitas ${ }^{3,4}$, and Luis Reis ${ }^{1,4}$ \\ ${ }^{1}$ Instituto Superior Técnico, Universidade de Lisboa, Av. Rovisco Pais, 1049-001 Lisbon, Portugal \\ ${ }^{2}$ Department of Design and Engineering, Faculty of Science and Technology, Bournemouth University, \\ Poole House, Talbot Campus, Fern Barrow, Poole BH12 5BB, UK \\ ${ }^{3}$ Atlântica, University School, Fabrica da Pólvora, Barcarena, 2730-036 Barcarena, Portugal \\ ${ }^{4}$ IDMEC, Instituto Superior Técnico, Av. Rovisco Pais, 1049-001 Lisbon, Portugal
}

\begin{abstract}
Fatigue studies of materials in simple or complex loading systems for any given lifetime is object of continuous research. This is due to the advancements on mechanical and structural components, as well as for new and innovative materials, which implies the knowledge of a materials response to all dynamic loads. The fatigue failure regime beyond what was once considered to be the fatigue limit (infinite life) is characterized between $10^{7}$ and $10^{9}$, known as Very High Cycle Fatigue regime. Due to the time consuming and wide energy consumption of conventional fatigue testing for such regime, fatigue tests under ultrasonic actuators are being used, capable of applying the dynamic loads at around $20 \mathrm{kHz}$. Nowadays, several variants of ultrasonic fatigue tests were already proposed and tested but it is still a somewhat limited fatigue test if compared to the conventional servo-hydraulic fatigue testing machines of general use. In this study, biaxial in plane stresses are induced in specially designed cruciform specimens with ultrasonic fatigue testing resonant principals. Two geometries were numerically analysed, manufactured and experimentally tested, the in-phase tension-tension (T-T) specimen and the out-of-phase compression-tension (C-T) specimen. All specific designed geometries go under a thorough numerical and several experiments analysis for their validation. The specimens showing a correct and as intended behaviour are led to failure.
\end{abstract}

\section{Introduction}

Many of every day mechanical devices and structures have many components subjected to dynamic and cyclic loadings, as cars, airplanes, trains, hydraulic machines and so on. To ensure the safety and reliability of the users and the machine every material employed to such components should be carefully chosen in accordance to the applied forces and life cycle. Therefore, the study of the materials resistance and behaviour to fatigue damage will ensure a higher safety and a correct projection of key components in such mechanical devices and structures. Many of the mentioned components can reach in their life time higher than $10^{7}$ cycles due to the high frequency of dynamic loads and/or long life in service. Due to the way

\footnotetext{
Corresponding author: luis.g.reis@ tecnico.ulisboa.pt
} 
the metals behave and the difficulty of testing a material with offer $10^{7}$ cycles it was once believed that for a certain stress or lower no fatigue failure could ever occur. Many researchers as Bathias and Sonsino have determined that no real fatigue limit is ever present $[1,2]$.

To study a material for any fatigue range of cycles and type of stresses, all specimens are designed carefully and machines capable of inducing high enough cyclic stress are chosen. In this study the fatigue regime of interest in the Very High Cycle Fatigue (VHCF) related between $10^{7}$ and $10^{9}$ cycles with induced transverse biaxial stresses using cruciform specimens. In order to study VHCF regime new fatigue machines were designed due to the very high number of cycles need to be induced to a specimen. A conventional machine can take several days just to reach $10^{7}$ cycles. Higher frequencies were needed, and so the ultrasonic fatigue testing machines were created. Such machines can induce cycling stresses with frequencies of around $20 \mathrm{kHz}$ or even higher. Such tests could only initially apply uniaxial tension-compression, bending and torsion tests [3-5].

Only a few multiaxial ultrasonic fatigue testing machines have been successfully conducted using the basics of the ultrasonic tests already designed [6, 7]. Such a machine capable of applying a biaxial state of stresses was created and is briefly described in this paper as well as a few results of tested specimens. All the tests were conducted with the base of the created machine by the laboratories of Instituto Superior Técnico [8].

In this research multiaxiality was achieved by creating aluminium cruciform specimens specially designed for the referenced machine. These specimens were carefully designed following an optimized cruciform geometry already employed and with the support of finite element analysis. Many different researches have already been made with aluminium as in $[9,10]$ as well as in the referenced biaxial machine by Palin-Luc et al. [6].

\section{Testing concept and methodology}

\subsection{Ultrasonic fatigue methodology}

Ultrasonic fatigue testing machines use resonant principals to achieve high enough stresses at a high frequency. In such machines, a piezoelectric transducer is used with a specially designed component's set, which are attached sequentially as: Transducer; Booster; Horn and finally the testing specimen. The components are specially designed to be exited in a specific resonant mode at the working frequency of the transducer. The used transducer has a frequency range of $20+/-0.5 \mathrm{kHz}$. The geometry of the Booster and Horn is designed to amplify the displacements provided from the transducer, while the specimen is designed to have a single and known area of high stress.

Taking as example the first ultrasonic test, tension-compression, the transducer transmits longitudinal displacements that are amplified by the booster and horn and subsequently transmitted to the material sample of interest for a given study. This sample, the specimen, is shaped as an hourglass and is excited in his first longitudinal resonant mode, which creates cyclic tension-compression stresses. The dimensions of the specimen are carefully chosen so that the resonant mode of interest and no other is as close to the middle range frequency of work of the transducer as possible.

\subsection{Cruciform specimen concept}

Following the mentioned ultrasonic fatigue concepts, two cruciform geometries were created with a specific resonant mode as close to $20 \mathrm{kHz}$ of frequency, an in-phase tension-tension (T-T) and an out-of-phase tension-compression (C-T). Both specimens were designed and evaluated with Finite element software taking in consideration a similar set as a uniaxial tension-compression ultrasonic fatigue test. Figure 1 shows a representation of the components set. 


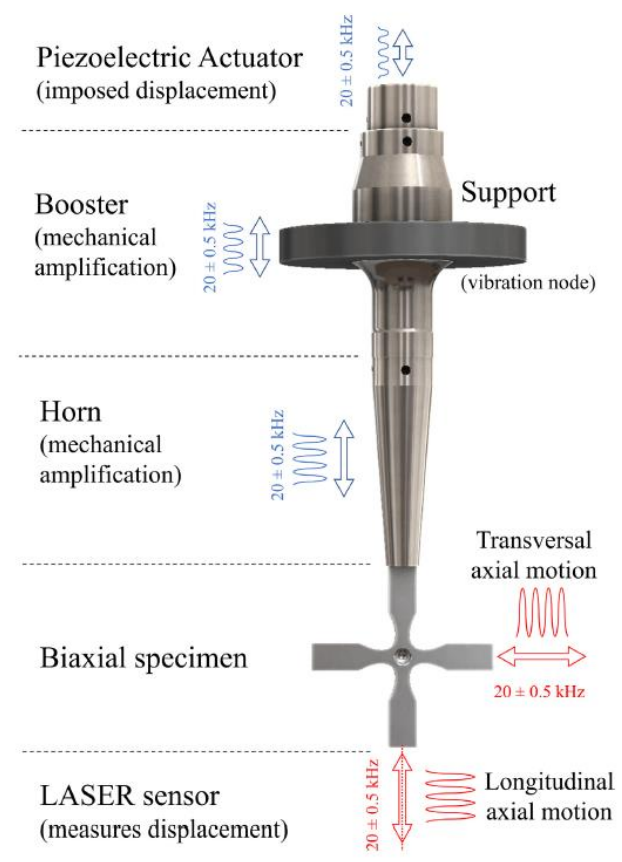

Fig. 1. Representation of the component's set for testing cruciform specimens

Each of the two specimens' dimensions resonant modes of interest have the highest stress is lowest in the connection and highest in the centre. The movement of the arms differs in phase between the specimens establishing the biaxiality as it can be observed in figure 2 .

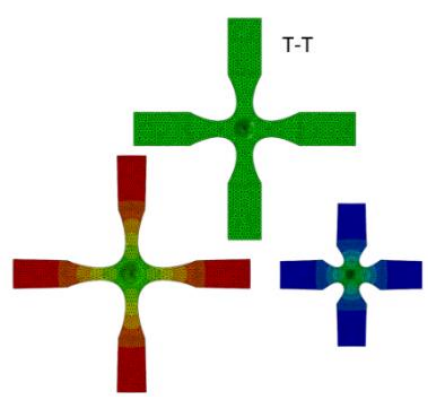

(A)

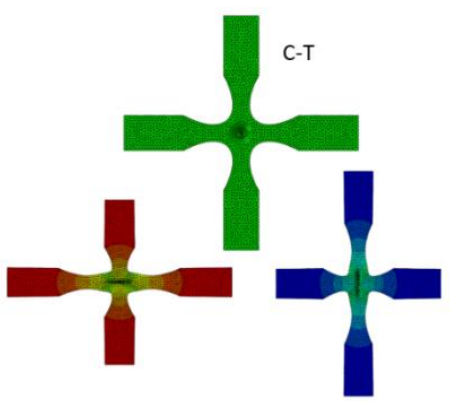

(B)

Fig. 2. Resonant displacement specimen representation: (A) in-phase T-T (B) out-of-phase C-T

The geometry and relation between all dimensions follow an optimized cruciform specimen presented by Baptista et all [11]. Several combinations were created and with the use of a finite element software, a modal analysis is made and the frequency for the resonant mode of interest. The first geometries for the T-T and C-T machined were followed by the literature research [12]. They are designed to have the biaxiality ratio $\left(B={ }^{\sigma}{ } / \sigma_{x}\right)$ of $B=1$ for T-T and $B=-1$ for $\mathrm{C}-\mathrm{T}$. 

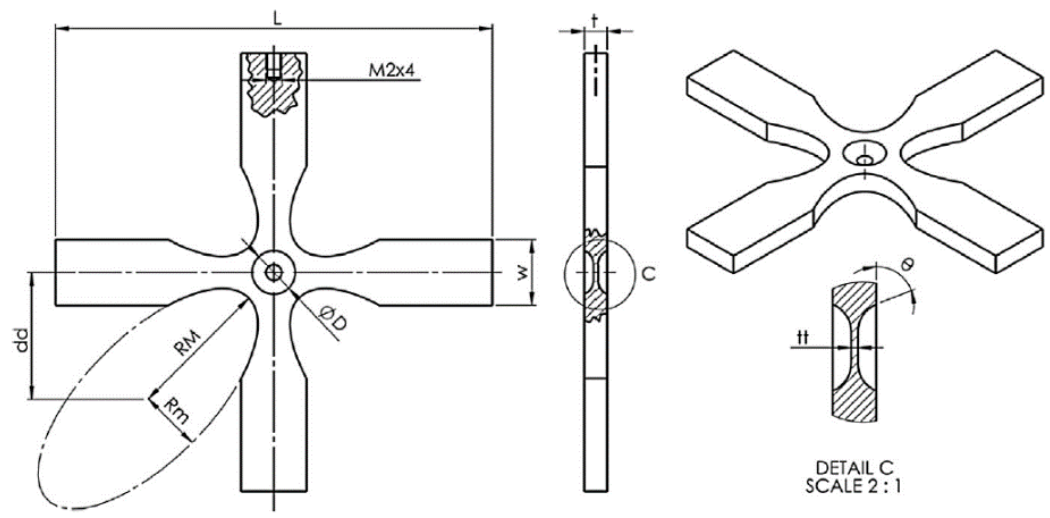

DETAILC
SCALE $2: 1$

Fig. 3. Cruciform geometry design and dimensions ( $\mathrm{mm})$ [12]

\subsection{Experimental procedure}

Several specimens of each type of cruciform specimens, T-T and C-T were analysed experimentally and tested to failure. Since the specimens are the first of its kind for the ultrasonic fatigue, and having a certain complexity, several test analyses were made to understand if the specimen's behaviour were as intended and predicted.

Firstly, a resonance analysis was made to see if they could even be excited within the working frequency of the transducer. Afterwards a Polytec Laser is pointed at several points of interest of the specimen and the difference of amplitude and phase are analysed.

All specimens were painted black in one side for better measurement of the temperature by a thermal camera. The regions of higher heat generation will be associated with higher deformation and thus higher stresses. Therefore, all specimens were analysed when under excitation using a thermal camera.

\section{Results}

The thermal images, as the one showed in figure 4, obtained during a test's strengthen the correct functioning of the $\mathrm{C}-\mathrm{T}$ specimen by showing to have a higher heat generation in the centre of the specimen where the stresses are the highest.

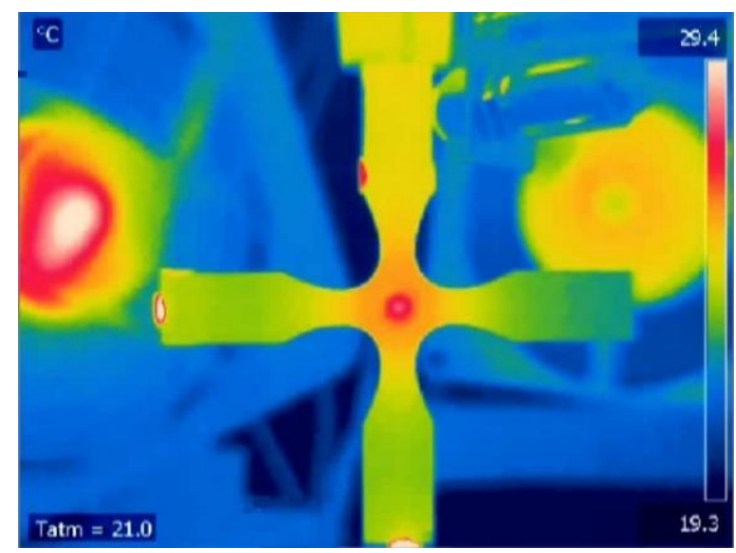

Fig. 4. Thermographic image showing the highest heat generation zone

The laser results have showed that the tested C-T specimens followed the displacement's relations as predicted, having the arms in out-of-phase condition and with no notable 
difference in amplitude and variance. Also, very little vertical movement was measured on the horizontal arms as well as horizontal in the vertical arms.

The failed specimen showed a crack near its centre and with angle of around 50 degrees to the horizontal arms, as it is presented in figure 5. The fracture surface was only visible after the specimen was put under a tensile machine.

(A)

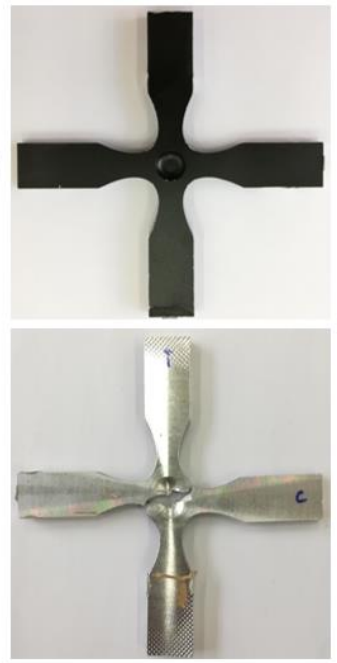

(C)

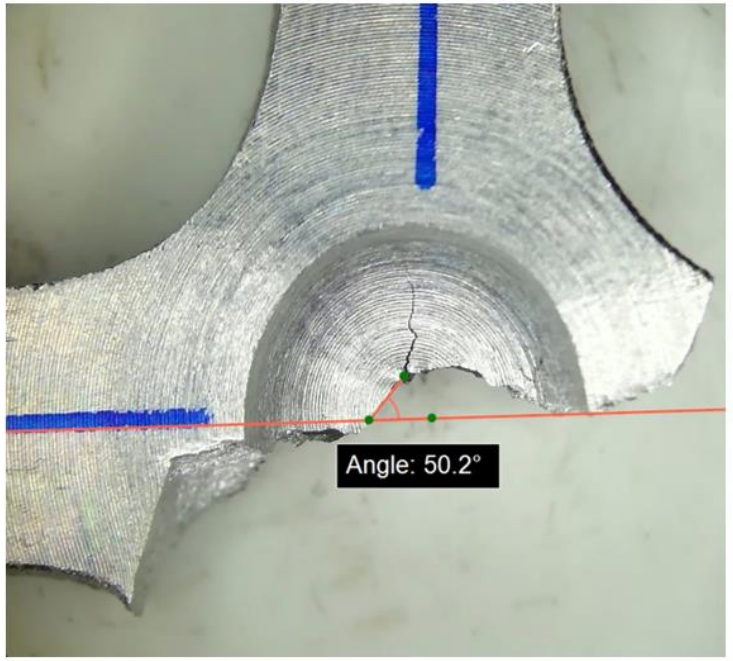

Fig. 5. (A) cruciform C-T painted black for thermographic imaging (B) C-T after total breakage in a tensile machine $(\mathrm{C})$ fatigue fracture angle

\section{Conclusion}

The experimental analysis showed that C-T specimen was working correctly. The correct functioning of the $\mathrm{C}$-T specimen was achieved by the laser measurements, the thermographic images and proved by the fact that it created a fatigue surface near its centre.

The crack showed to have around $\pm 50^{\circ}$ degrees to the horizontal arms. This angle is as predicted by most fatigue damage parameters calculated by Batista et all [13] for the optimized geometry followed in this study within the HCF regime. This emphasize the correct stress induction of the C-T specimen. All tested T-T specimens did not work as intended even thought they could be excited by the transducer. The excited mode exhibited a displacement combination of modes with the mode of interest. The FEA analysis proved that for the tested specimen there were resonant modes with similar frequencies and consequently the experimental results showed that such influence changes the specimen behaviour in such a way making it unreliable for the axial-axial in-phase fatigue test. It should be remarked that a different geometry is under development to shift the close resonant modes of non-interest for the frequency of work $20 \pm 0.5 \mathrm{kHz}$ just as the C-T specimen, which should correct the T-T resonant mode.

The authors fully acknowledge the support and financing provided by FCT for the Multiaxial Very High Cycle Fatigue project PTDC/EMS-PRO/5760/2014

This work was also supported by FCT, through IDMEC, under LAETA, project UID/EMS/50022/2019.

\section{References}

[1] C. Bathias, There is no infinite fatigue life in metallic materials, Fatigue Fract. Eng. Mater. Struct., 22, 559-565, (1999).

[2] C. M. Sonsino, Course of SN-curves especially in the high-cycle fatigue regime with 
regard to component design and safety, Int. J. Fatigue, 29, 2246-2258, (2007).

[3] C. Bathias, Piezoelectric fatigue testing machines and devices, Int. J. Fatigue, 28, 11, 1438-1445, (2006).

[4] H. Soares, P. Costa, M. Freitas, and L. Reis, Fatigue life assessment of a railway wheel material under HCF and VHCF conditions, MATEC Web Conf., 165, 09003, (2018).

[5] H. Q. Xue, H. Tao, F. Montembault, Q. Y. Wang, and C. Bathias, Development of a three-point bending fatigue testing methodology at $20 \mathrm{kHz}$ frequency, Int. J. Fatigue, 29, 2085-2093, (2007).

[6] C. Brugger, T. Palin-Luc, P. Osmond, and M. Blanc, Gigacycle fatigue behavior of a cast aluminum alloy under biaxial bending: experiments with a new piezoelectric fatigue testing device, 21st Eur. Conf. Fract. ECF21, 2, 96-100, (2016).

[7] P. Costa, M. Vieira, L. Reis, A. Ribeiro, and M. de Freitas, New specimen and horn design for combined tension and torsion ultrasonic fatigue testing in the very high cycle fatigue regime, Int. J. Fatigue, 103, 248-257, (2017).

[8] M. Vieira, M. De Freitas, L. Reis, A. M. R. Ribeiro, and M. Da Fonte, Development of a very high cycle fatigue (VHCF) multiaxial testing device, Frat. ed Integrita Strutt., 10, 37, 131-137, (2016).

[9] M. Cremer, M. Zimmermann, and H. J. Christ, High-frequency cyclic testing of welded aluminium alloy joints in the region of very high cycle fatigue (VHCF), Int. J. Fatigue, 57, 120-130, (2013).

[10] Q. Y. Wang, T. Lib, and X. G. Zenga, Gigacycle fatigue behavior of high strength aluminum alloys, Procedia Eng., 2, 1, 65-70, (2010).

[11] R. Baptista, R. A. Cláudio, L. Reis, J. F. A. Madeira, and M. Freitas, Numerical study of in-plane biaxial fatigue crack growth with different phase shift angle loadings on optimal specimen geometries, Theor. Appl. Fract. Mech., 85, 16-25, (2016).

[12] D. Montalvão and A. Wren, Redesigning axial-axial (biaxial) cruciform specimens for very high cycle fatigue ultrasonic testing machines, Heliyon, 3, 11, e00466, (2017).

[13] R. Baptista, R. A. Cláudio, L. Reis, J. F. A. Madeira, and M. Freitas, Numerical study of fatigue crack initiation and propagation on optimally designed cruciform specimens, Procedia Struct. Integr., 1, 98-105, (2016). 